\title{
Torsional Vibrations of Circular Elastic Plates with Thickness Steps
}

\author{
Min K. Kang, Rui Huang, and Terence Knowles, Member, IEEE
}

\begin{abstract}
This paper presents a theoretical study of torsional vibrations in isotropic elastic plates. The exact solutions for torsional vibrations in circular and annular plates are first reviewed. Then, an approximate method is developed to analyze torsional vibrations of circular plates with thickness steps. The method is based on an approximate plate theory for torsional vibrations derived from the variational principle following Mindlin's series expansion method. Approximate solutions for the zeroth- and first-order torsional modes in the circular plate with one thickness step are presented. It is found that, within a narrow frequency range, the first-order torsional modes can be trapped in the inner region where the thickness exceeds that of the outer region. The mode shapes clearly show that both the displacement and the stress amplitudes decay exponentially away from the thickness step. The existence and the number of the trapped first-order torsional modes in a circular mesa on an infinite plate are determined as functions of the normalized geometric parameters, which may serve as a guide for designing distributed torsionalmode resonators for sensing applications. Comparisons between the theoretical predictions and experimental measurements show close agreements in the resonance frequencies of trapped torsional modes.
\end{abstract}

\section{INTRODUCTION}

$\mathrm{T}$ ORSIONAL vibrations of circular plates and cylindrical rods have been studied for many years. Recent developments of torsional-mode sensors and actuators in microand nanoelectromechanical systems have renewed interests in this topic [1]-[5]. Previous studies of torsional vibrations have focused largely on cylindrical rods or shafts, for which a one-dimensional mathematical model based on the "strength-of-materials" approach [6] has been widely used in practice. However, it has been shown that the one-dimensional model is only accurate at low frequencies, and three-dimensional analysis is required at high frequencies [7]. In particular, shafts with stepped cross sections are common in engineering structures. Several three-dimensional methods have been developed to analyze torsional vibrations of stepped shafts [7]-[11]. Johnson et al. [11] showed that, within a certain frequency range, torsional modes can be trapped in the central section of stepped solid cylinders with a slightly larger diameter such that the vibration amplitude decays exponentially with the

Manuscript received July 1, 2005; accepted July 14, 2005.

M. K. Kang and R. Huang are with the Research Center for Mechanics of Solids, Structures and Materials, Department of Aerospace Engineering and Engineering Mechanics, The University of Texas, Austin, TX 78712 (e-mail: ruihuang@mail.utexas.edu).

T. Knowles is with Texzec, Inc., Round Rock, TX 78681. distance from the central section. Such trapped modes may find interesting applications in the design of resonators, transducers, and sensors.

In a parallel development, trapped vibrations in plates have been studied for many decades to improve the performance of quartz crystal resonators. By mass loading of quartz crystal plates with electrodes, thickness-shear vibrations can be trapped under the electrodes with amplitude decreasing exponentially away from the electrodes in the unplated region [12]-[16]. It also has been observed that, by decreasing the plate thickness from center to edge or contouring, the thickness-shear vibrations can be confined in the center portion of the plate, which improves the resonator performance by reducing edge leakage due to boundary mismatch and mode conversion [17]-[19]. Much less attention has been paid to torsional vibrations of plates. By using Love's thin plate theory [20], Onoe [21] analyzed the contour vibrations of circular plates, including torsional modes (Onoe used the term "tangential modes"). The result was used by Meitzler [22] in an ultrasonic technique for determining elastic constants of glass wafers. For thick plates (thickness comparable to radius), exact solutions are available for both solid circular plates and annular plates, which are essentially the same as the exact solutions to the classical Pochhammer equation for cylindrical rods and hollow cylinders [23], [24]. For plates with nonuniform thickness, an exact analytical solution generally is not possible [25]. This paper develops an approximate method for analyzing torsional vibrations of circular plates with thickness varying in the radial direction as steps [Fig. 1(c)]. Such plates are of interest because torsional vibrations may be trapped near the steps, similar to that in stepped solid cylinders [11] and trapping of thickness-shear vibrations in contoured plates [17]-[19]. Recent experiments by Knowles et al. [26] have observed trapped torsional modes in both stepped and contoured aluminum plates, which may be used to design sensors with improved performance in the presence of liquids.

This paper is organized as follows. Section II reviews the general three-dimensional theory and exact solutions for torsional vibrations of circular and annular plates with uniform thickness [Fig. 1(a) and (b)]. Section III develops an approximate plate theory from variational principle. In Section IV, an approximate method is developed for torsional vibrations of plates with thickness steps [Fig. 1(c)], and approximate solutions are obtained for the zeroth- and first-order modes. Trapped first-order modes are identified. A diagram is constructed predicting the existence and the number of trapped torsional modes in a circular mesa on 


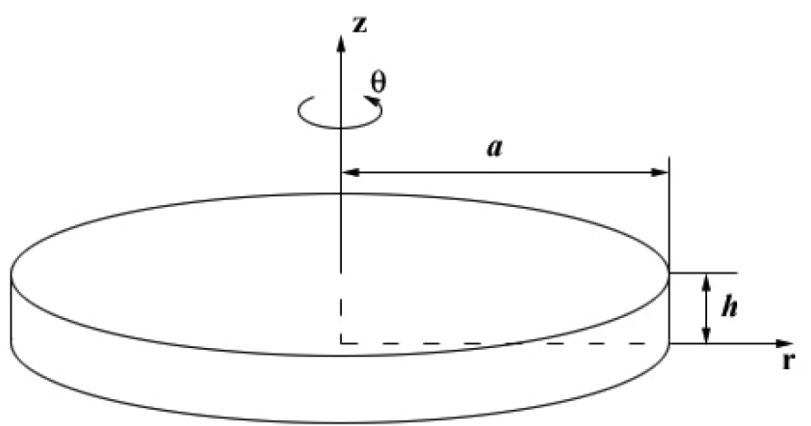

(a)

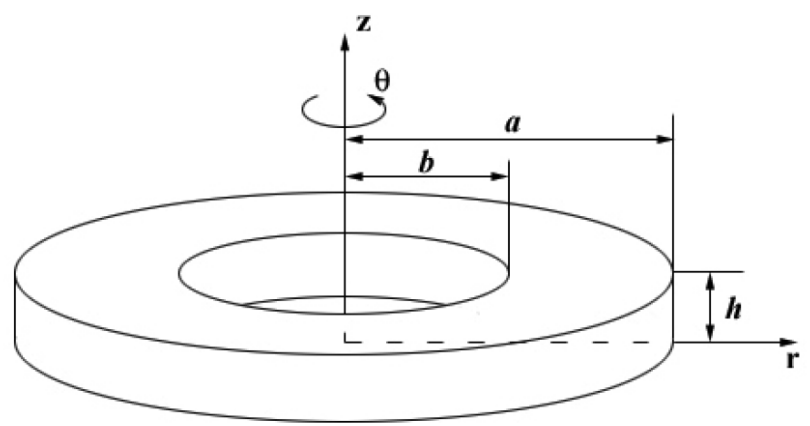

(b)

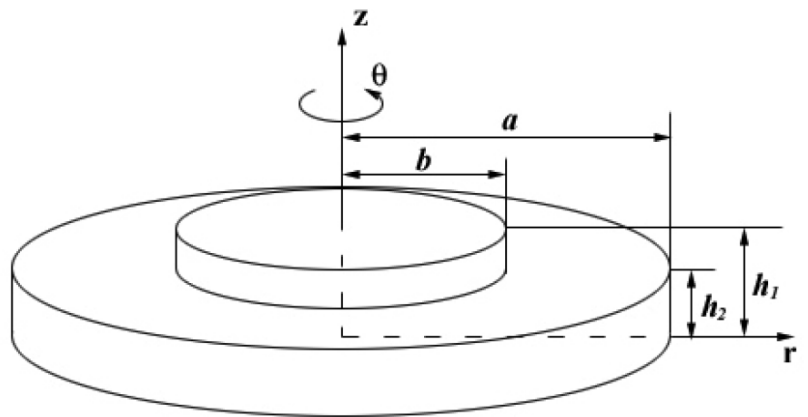

(c)

Fig. 1. Schematics of the plates. (a) A uniform circular plate. (b) An annular plate. (c) A circular plate with a thickness step.

an infinite plate. The theoretical results are compared to experimental measurements. Section V concludes with remarks on potential applications of the theoretical results.

\section{General Theory and Exact Solutions}

Fig. 1 illustrates the geometries of the plates considered in this paper. Assuming axisymmetric motion and isotropic, linear elastic materials, the momentum equation for the torsional motion is:

$$
\frac{\partial^{2} u_{\theta}}{\partial r^{2}}+\frac{1}{r} \frac{\partial u_{\theta}}{\partial r}-\frac{u_{\theta}}{r^{2}}+\frac{\partial^{2} u_{\theta}}{\partial z^{2}}=\frac{\rho}{\mu} \frac{\partial^{2} u_{\theta}}{\partial t^{2}}
$$

where $r, \theta, z$ are the cylindrical coordinates as defined in Fig. $1, t$ is the time, $u_{\theta}$ is the torsional displacement, $\rho$ is the mass density, and $\mu$ is the shear modulus.

The stress associated with the torsional motion has two nonzero shear components, which relate to the torsional displacement by:

$$
\begin{aligned}
\sigma_{z \theta} & =\mu \frac{\partial u_{\theta}}{\partial z}, \\
\sigma_{r \theta} & =\mu\left(\frac{\partial u_{\theta}}{\partial r}-\frac{u_{\theta}}{r}\right) .
\end{aligned}
$$

For plates with uniform thickness, (1) can be solved by separation of variables. Assume a harmonic solution, namely:

$$
u_{\theta}(r, z, t)=P(r) Q(z) e^{i \omega t},
$$

where $\omega$ is the angular frequency of vibration. Substitution of (4) into (1) leads to:

$$
\begin{aligned}
& \frac{d^{2} P}{d r^{2}}+\frac{1}{r} \frac{d P}{d r}+\left(\beta^{2}-\frac{1}{r^{2}}\right) P=0, \\
& \frac{d^{2} Q}{d z^{2}}+k^{2} Q=0
\end{aligned}
$$

where:

$$
\beta^{2}+k^{2}=\frac{\rho \omega^{2}}{\mu}
$$

For $\beta, k \neq 0$, the general solutions to (5) and (6) are:

$$
\begin{aligned}
& P(r)=A J_{1}(\beta r)+B Y_{1}(\beta r), \\
& Q(z)=C \sin (k z)+D \cos (k z),
\end{aligned}
$$

where $J_{1}$ and $Y_{1}$ are the first-order Bessel functions of the first and second kinds, respectively, and the coefficients $A$, $B, C, D$ must be determined from boundary conditions. An alternative form of the solution (8) is:

$$
P(r)=A^{\prime} H_{1}^{(1)}(\beta r)+B^{\prime} H_{1}^{(2)}(\beta r),
$$

where $H_{1}^{(1)}$ and $H_{1}^{(2)}$ are the first-order Hankel functions of the first and second kinds. Due to their asymptotic behavior, the Hankel functions sometimes offer convenience for physical interpretations of the solution.

When $\beta=0$, the solution (8) is replaced by a special solution:

$$
P(r)=A^{*} r+\frac{B^{*}}{r}
$$

Similarly, when $k=0$, the solution (9) is replaced by:

$$
Q(z)=C^{*} z+D^{*}
$$

A complete solution for free vibrations can be obtained by a superposition of all possible solutions in the form of (4) with the value of $\beta$ or $k$ determined from the boundary condition. For forced vibrations, a particular solution satisfying the forcing boundary conditions must be included in the superposition. This paper focuses on free vibrations only. 


\section{A. Free Vibrations of a Circular Plate}

First consider a circular plate of radius $a$ and thickness $h$ with traction-free boundaries at all surfaces and edges [Fig. 1(a)]. The boundary condition requires that:

$$
\begin{aligned}
& \frac{d P}{d r}-\frac{P}{r}=0 \quad \text { at } r=a, \\
& \frac{d Q}{d z}=0 \quad \text { at } z=0 \text { and } h .
\end{aligned}
$$

In addition, it is implied that the displacement at the center of the plate $(r=0)$ is finite. Consequently, any singular terms with respect to $r$ must be discarded from the solution. Therefore, the solutions become:

$$
P_{m}(r)=\left\{\begin{array}{ll}
A_{0} r & m=0 \\
A_{m} J_{1}\left(\beta_{m} r\right) & m=1,2, \cdots
\end{array},\right.
$$

where $\beta_{m}=s_{m} / a$ and $s_{m}$ is the $m$ th nonzero root to $J_{2}(s)=0$, and:

$$
Q_{n}(z)=D_{z} \cos \left(k_{n} z\right),
$$

where $k_{n}=(n \pi / h)$ for $n=0,1,2, \ldots$

Combining (15) and (16), one obtains the complete solution for torsional vibrations of the circular plate, namely:

$$
\begin{aligned}
u_{\theta}(r, z, t)= & \sum_{n=0}^{\infty}\left[A_{n} r \cos \left(k_{n} z\right) e^{i \omega_{n} t}\right. \\
& \left.+\sum_{m=1}^{\infty} B_{m n} J_{1}\left(\beta_{m} r\right) \cos \left(k_{n} z\right) e^{i \omega_{m n} t}\right],
\end{aligned}
$$

where:

$$
\begin{aligned}
\omega_{n} & =\frac{n \pi}{h} \sqrt{\frac{\mu}{\rho}} \\
\omega_{m n} & =\sqrt{\frac{\mu}{\rho}\left(\frac{n^{2} \pi^{2}}{h^{2}}+\frac{s_{m}^{2}}{a^{2}}\right)} .
\end{aligned}
$$

Solution (17) consists of all the possible torsional modes that satisfy the boundary conditions for the circular plate, where (18) and (19) give the corresponding resonant frequency of each mode.

Fig. 2 shows the spectrum for torsional vibrations of circular plates, where the frequency is normalized by the first cut-off frequency, $\omega_{1}=(\pi / h) \sqrt{\mu / \rho}$, and plotted against the ratio between the thickness and the radius of the plate. The spectrum includes two limiting cases. To the right end of the spectrum, the first several modes with the lowest frequencies correspond to the case with $\beta=0$. In this case, the displacement is proportional to the $r$-coordinate as given by the first term in the bracket of (17), and the resonance frequencies $\left(\omega_{n}\right)$ are independent of the radius of the plate. These modes are identical to those predicted by the one-dimensional model for long cylindrical rods. To the left end of the spectrum, the first several modes with the lowest frequencies now correspond to the case with

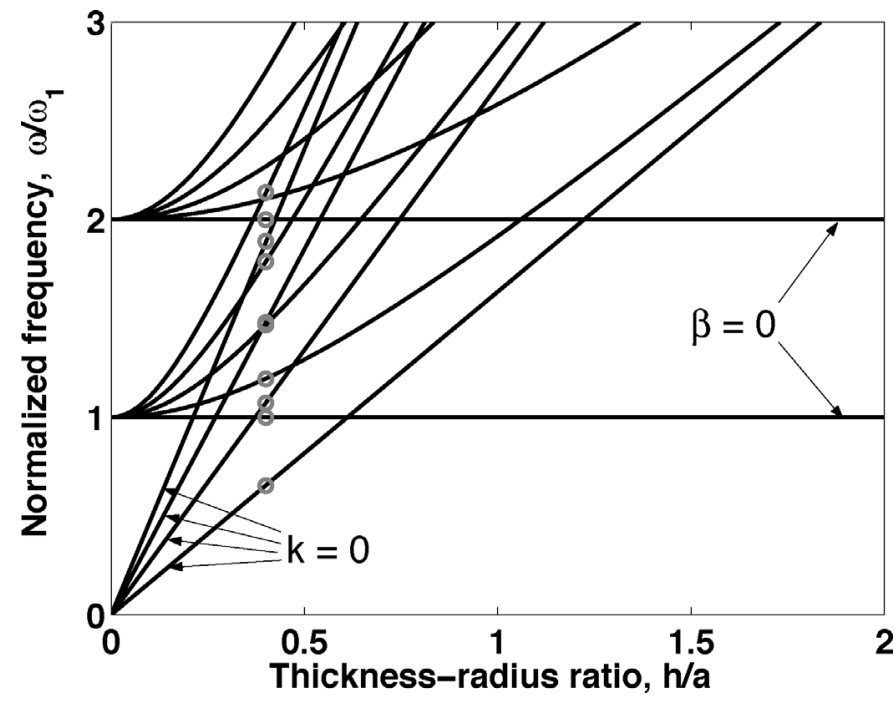

Fig. 2. Spectrum for torsional vibrations of uniform circular plates. The circles are numerical results from Zhou et al. [27].

$k=0(n=0)$, for which the displacement is independent of $z$ and the resonance frequency is independent of the thickness $h$. These modes are identical to those predicted by Onoe [21] for thin circular plates $(h / a \ll 1)$. Between the two limits, the frequencies from different groups are interwoven. The spectrum clearly shows when the onedimensional model or the thin-plate approximations can be used and when the exact three-dimensional analysis is required. Zhou et al. [27] recently conducted a threedimensional vibration analysis using the Chebyshev-Ritz method and predicted the first 10 resonance frequencies for a circular plate with thickness ratio $h / a=0.4$, as shown by the circles in Fig. 2. The excellent agreement confirms the accuracy of the Chebyshev-Ritz method.

\section{B. Free Vibrations of Annular Plates}

Next consider an annular plate with outer radius $a$, inner radius $b$, and thickness $h$ [Fig. 1(b)]. Both inner and outer edges are traction free. In addition to the boundary conditions (13) and (14), the traction-free condition at the inner edge requires that:

$$
\frac{d P}{d r}-\frac{P}{r}=0 \text { at } r=b .
$$

Consequently, (15) becomes:

$$
P_{m}(r)=\left\{\begin{array}{ll}
A_{0} r & m=0 \\
A_{m}\left[Y_{2}\left(\beta_{m} a\right) J_{1}\left(\beta_{m} r\right)\right. & \\
\left.-J_{2}\left(\beta_{m} a\right) Y_{1}\left(\beta_{m} r\right)\right] & m=1,2, \cdots
\end{array},\right.
$$

where $\beta_{m}=s_{m} / a$ with $s_{m}$ being the $m$ th root to the following equation:

$$
J_{2}(s) Y_{2}(\eta s)-J_{2}(\eta s) Y_{2}(s)=0,
$$

and $\eta=b / a$. When the inner radius $b=0,(21)$ recovers (15). 


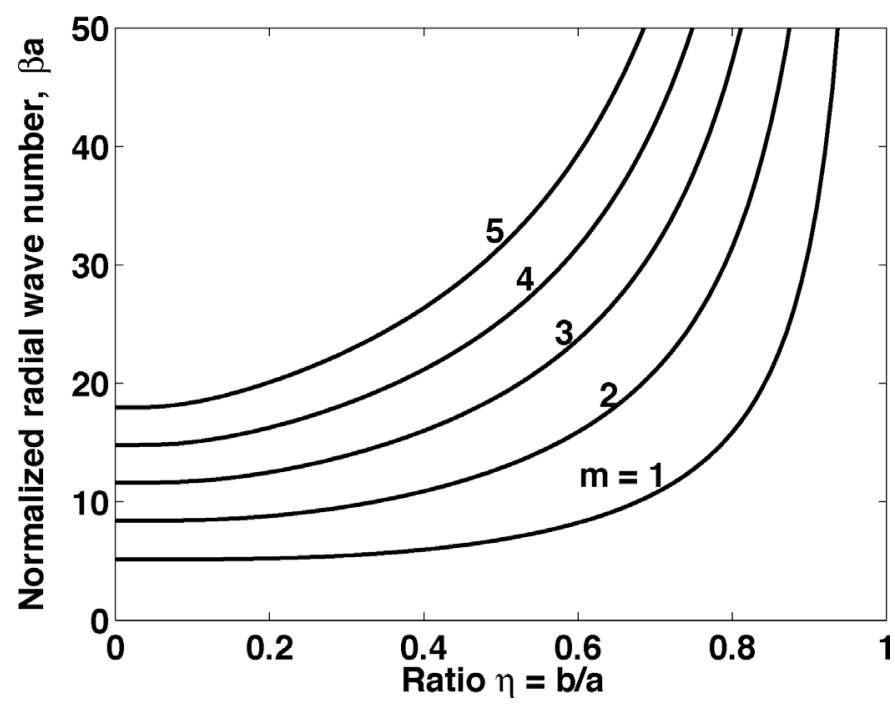

Fig. 3. Radial wave number for torsional vibrations of annular plates versus the ratio between inner and outer radius.

With (16) unchanged, the complete solution for torsional vibrations of the annular plate is:

$$
\begin{aligned}
u_{\theta}(r, z, t)= & \sum_{n=0}^{\infty}\left[A_{n} r \cos \left(k_{n} z\right) e^{i \omega_{n} t}\right. \\
& +\sum_{m=1}^{\infty} B_{m n}\left(Y_{2}\left(\beta_{m} a\right) J_{1}\left(\beta_{m} r\right)\right. \\
& \left.\left.-J_{2}\left(\beta_{m} a\right) Y_{1}\left(\beta_{m} r\right)\right) \cos \left(k_{n} z\right) e^{i \omega_{m n} t}\right],
\end{aligned}
$$

where the frequencies $\omega_{n}$ and $\omega_{m n}$ take the same form as in (18) and (19).

The frequency spectrum for the annular plates is similar to Fig. 2, but the radial wave number $\beta_{m}$ varies with $\eta$, the ratio between the inner radius and the outer radius. Fig. 3 shows the first five radial wave numbers obtained from (22) as functions of the ratio. The left end of the figure corresponds to the case of solid circular plates $(\eta=$ 0 ). Toward the right end, the wave numbers rise rapidly, which correspond to thin-walled tubes. A similar result was obtained by Clark [23] for hollow cylinders.

Following similar procedures, exact solutions for torsional vibrations of circular and annular plates with other boundary conditions can be obtained. For plates with nonuniform thickness, however, exact solutions generally are not available [25]. Consider a circular plate with a thickness step [Fig. 1(c)], which has thickness $h_{1}$ at the inner region $(0<r<b)$ and thickness $h_{2}\left(h_{2} \neq h_{1}\right)$ at the outer region $(b<r<a)$. The general solution for torsional vibrations of such plates should consist of two parts. For the inner region, the displacement takes the form of (17); for the outer region, the displacement takes the form of (23). The two parts are coupled through the joint boundary at $r=b$, where the continuity conditions for the displacement and the shear traction have to be applied. However, exact solutions satisfying the continuity conditions cannot be obtained analytically. The remain- der of this paper develops an approximate plate theory, from which approximate solutions to torsional vibrations of circular plates with thickness steps are obtained.

\section{Approximate Plate Theory}

A general procedure for deducing approximate equations for elastic plates from the three-dimensional theory of elasticity was first introduced by Mindlin [28] based on the series expansion methods and the variational method. The procedure has been used to derive approximate plate theories for both elastic and piezoelectric crystal plates with uniform [29]-[31] and nonuniform thickness [18], [19], $[32]$. Here we follow the same procedure to derive approximate equations for torsional vibrations of isotropic elastic plates. The approximate equations then will be used to deduce an approximate solution to torsional vibrations of circular plates with thickness steps in the next section.

Considering torsional motion of a linear elastic continuum of volume $V$ bounded by a surface $S$, the variational principle leads to:

$$
\begin{aligned}
& \int d t \int_{V}\left(\frac{\partial \sigma_{r \theta}}{\partial r}+\frac{\partial \sigma_{z \theta}}{\partial z}+\frac{2 \sigma_{r \theta}}{r}-\rho \frac{\partial^{2} u_{\theta}}{\partial t^{2}}\right) \delta u_{\theta} d V=0 \\
& \int d t \int_{S}\left(t_{\theta}-n_{r} \sigma_{r \theta}-n_{z} \sigma_{z \theta}\right) \delta u_{\theta} d S=0
\end{aligned}
$$

where $t_{\theta}$ is the traction at boundary $S$, and $\mathbf{n}$ is the outward normal at the boundary. For a circular plate [Fig. 1(a)], the boundary $S$ consists of two surfaces at $z=0$ and $h$ and the edge face at $r=a$. For an annular plate [Fig. 1(b)], another edge at $r=b$ adds to the boundary.

Following Mindlin [28], the displacement may be expanded into a series of functions of the thickness coordinate. Various function series have been used to develop approximate plate theories. For torsional vibrations, noticing the form of exact solutions in (17) and (23), we expand the displacement into a cosine series:

$$
u_{\theta}(r, z, t)=\sum_{n=0}^{\infty} u_{\theta}^{(n)}(r, t) \cos \left(n \pi \frac{z}{h}\right) .
$$

The orthogonality of the cosine series leads to:

$$
u_{\theta}^{(n)}(r, t)=\frac{2-\delta_{n 0}}{h} \int_{0}^{h} u_{\theta}(r, z, t) \cos \left(n \pi \frac{z}{h}\right) d z
$$

where $\delta_{m n}=1$ if $m=n$ and $\delta_{m n}=0$ otherwise.

Substituting (26) into (24) and integrating over the thickness of the plate, we obtain that:

$$
\begin{aligned}
\int d t \int_{A} \sum_{n=0}^{\infty} & {\left[\sigma_{r \theta, r}^{(n)}+\frac{2}{r} \sigma_{r \theta}^{(n)}+\frac{n \pi}{h} \bar{\sigma}_{z \theta}^{(n)}\right.} \\
+ & \left.\frac{1}{h} F_{\theta}^{(n)}-\frac{1+\delta_{n 0}}{2} \rho \ddot{u}_{\theta}^{(n)}\right] \delta u_{\theta}^{(n)} d A=0,
\end{aligned}
$$


where $A$ is a plane parallel to the surface of the plate, and:

$$
\begin{aligned}
\sigma_{r \theta}^{(n)} & =\frac{1}{h} \int_{0}^{h} \sigma_{r \theta} \cos \left(n \pi \frac{z}{h}\right) d z, \\
\bar{\sigma}_{z \theta}^{(n)} & =\frac{1}{h} \int_{0}^{h} \sigma_{z \theta} \sin \left(n \pi \frac{z}{h}\right) d z, \\
F_{\theta}^{(n)} & =(-1)^{n} \sigma_{z \theta}(h)-\sigma_{z \theta}(0) .
\end{aligned}
$$

In a similar manner, substituting (26) into (25), we obtain that:

$$
\int d t \int_{C} \sum_{n=0}^{\infty}\left[t_{\theta}^{(n)}-n_{r} \sigma_{r \theta}^{(n)}\right] \delta u_{\theta}^{(n)} d s=0,
$$

where $C$ is the contour of the plate edge(s), and:

$$
t_{\theta}^{(n)}=\frac{1}{h} \int_{0}^{h} t_{\theta} \cos \left(n \pi \frac{z}{h}\right) d z .
$$

In deriving (32), the integral over the two surfaces of the plate has been set zero as the surface traction is specified through the definition of $F_{\theta}^{(n)}$ in (31).

For (28) and (32) to be true for arbitrary variations, we have, in $A$ :

$$
\sigma_{r \theta, r}^{(n)}+\frac{2}{r} \sigma_{r \theta}^{(n)}+\frac{n \pi}{h} \bar{\sigma}_{z \theta}^{(n)}+\frac{1}{h} F_{\theta}^{(n)}=\frac{1+\delta_{n 0}}{2} \rho \ddot{u}_{\theta}^{(n)},
$$

and the boundary condition on $C$ :

$$
t_{\theta}^{(n)}=n_{r} \sigma_{r \theta}^{(n)} \text { or } u_{\theta}^{(n)}=\hat{u}_{\theta}^{(n)},
$$

where $\hat{u}_{\theta}^{(n)}$ is any specified displacement at the edge. As a result, the three-dimensional problem described by (24) and (25) has been transformed to a system of twodimensional equations. The variables, $u_{\theta}^{(n)}, \sigma_{r \theta}^{(n)}, \bar{\sigma}_{z \theta}^{(n)}$, $F_{\theta}^{(n)}, t_{\theta}^{(n)}$ are the $n$ th-order, two-dimensional components of displacement, shear stresses, face traction, and edge traction, respectively.

By inserting (26) into (2) and (3) and then into (29) and (30), we obtain that:

$$
\begin{aligned}
& \sigma_{r \theta}^{(n)}=\mu \frac{1+\delta_{n 0}}{2}\left[\frac{\partial u_{\theta}^{(n)}}{\partial r}-\frac{u_{\theta}^{(n)}}{r}\right], \\
& \bar{\sigma}_{z \theta}^{(n)}=-\mu \frac{n \pi}{2} \frac{u_{\theta}^{(n)}}{h} .
\end{aligned}
$$

Substitution of (36) and (37) into (34) leads to:

$$
\begin{aligned}
\mu\left[\frac{\partial^{2} u_{\theta}^{(n)}}{\partial r^{2}}\right. & \left.+\frac{1}{r} \frac{\partial u_{\theta}^{(n)}}{\partial r}-\frac{u_{\theta}^{(n)}}{r^{2}}\right] \\
& -\mu\left(\frac{n \pi}{h}\right)^{2} u_{\theta}^{(n)}+\frac{2-\delta_{n 0}}{h} F_{\theta}^{(n)}=\rho \ddot{u}_{\theta}^{(n)},
\end{aligned}
$$

which is the $n$ th-order displacement equation of torsional motion.
Each specific term in the infinite series expansion of the displacement in (26) can be obtained by solving (38) with associated boundary conditions at the edge(s) given by (35). For circular plates and annular plates with tractionfree surfaces $\left(F_{\theta}^{(n)}=0\right)$, the infinite series expansion is identical to the exact solutions in (17) and (23). For circular plates with thickness steps [Fig. 1(c)], specific displacement terms for the inner and outer regions can be obtained from (38) separately, and the continuity condition at the step can be approximated by (35). The procedure is presented in Section IV.

\section{Approximate Solution for Stepped Plates}

Fig. 1(c) sketches the geometry of the plate under consideration. In general, all orders of the displacement in the series expansion must be considered for both the inner and outer regions of the plate, which leads to an infinite number of coupled equations. Such analysis, however, is practically impossible. Instead, as generally understood, at one resonance frequency, one particular displacement component dominates the others. Consequently, it is possible to analyze a single mode approximately by neglecting the coupling with the other modes. Similar approaches have been widely used in the truncation procedures in the development of approximate plate theories [28]-[32]. The present study focuses on the vibration of the inner region, which may be trapped by the step. As an approximation, the displacement components of various orders in the inner region are considered separately. The displacement of the outer region is expanded into a series to ensure the displacement continuity at the junction, which will be further truncated into a finite number of terms. Therefore, for the $n$ th-order torsional vibration of the inner region, the displacement of the stepped plate takes the form of (39) (see next page).

Solving (38) for the inner and outer regions separately, we obtain that:

$$
\begin{aligned}
u_{\theta 1}^{(n)} & =A_{1}^{(n)} J_{1}\left(\beta_{1}^{(n)} r\right) e^{i \omega t}, \\
u_{\theta 2}^{(m)} & =\left[A_{2}^{(m)} J_{1}\left(\beta_{2}^{(m)} r\right)+B_{2}^{(m)} Y_{1}\left(\beta_{2}^{(m)} r\right)\right] e^{i \omega t},
\end{aligned}
$$

where:

$$
\begin{aligned}
& \beta_{1}^{(n)^{2}}=\frac{\rho}{\mu} \omega^{2}-\left(\frac{n \pi}{h_{1}}\right)^{2}, \\
& \beta_{2}^{(m)^{2}}=\frac{\rho}{\mu} \omega^{2}-\left(\frac{m \pi}{h_{2}}\right)^{2} .
\end{aligned}
$$

The corresponding in-plane shear stresses are:

$$
\begin{aligned}
\sigma_{r \theta 1}^{(n)}= & -\frac{1+\delta_{n \theta}}{2} \mu A_{1}^{(n)} \beta_{1}^{(n)} J_{2}\left(\beta_{1}^{(n)} r\right) e^{i \omega t}, \\
\sigma_{r \theta 2}^{(m)}= & -\frac{1+\delta_{m 0}}{2} \mu \beta_{2}^{(m)}\left[A_{2}^{(m)} J_{2}\left(\beta_{2}^{(m)} r\right)\right. \\
& \left.+B_{2}^{(m)} Y_{2}\left(\beta_{2}^{(m)} r\right)\right] e^{i \omega t} .
\end{aligned}
$$




$$
u_{\theta}(r, z, t)= \begin{cases}u_{\theta 1}^{(n)}(r, t) \cos \left(n \pi \frac{z}{h_{1}}\right), & r<b \text { and } 0<z<h_{1} ; \\ \sum_{m=0}^{\infty} u_{\theta 2}^{(m)}(r, t) \cos \left(m \pi \frac{z}{h_{2}}\right), & b<r<a \text { and } 0<z<h_{2} .\end{cases}
$$

At the junction $(r=b)$, both the displacement and the traction are required to be continuous. In the case of $h_{1}>h_{2}$, part of the edge of the inner region is traction free with unspecified displacement. Thus, the continuity of the displacement is only required for $0<z<h_{2}$, which, by (27), leads to:

$$
u_{\theta 2}^{(m)}=\frac{2-\delta_{m 0}}{h_{2}} \int_{0}^{h_{2}} u_{\theta 1}^{(n)} \cos \left(n \pi \frac{z}{h_{1}}\right) \cos \left(m \pi \frac{z}{h_{2}}\right) d z
$$

However, the traction at the edge of the inner region is fully specified by the traction-free part and the continuous part, which requires that:

$$
\begin{gathered}
\sigma_{r \theta 1}^{(n)}= \\
\frac{1}{h_{1}} \int_{0}^{h_{2}} \sum_{m=0}^{\infty}\left(2-\delta_{m 0}\right) \sigma_{r \theta 2}^{(m)} \cos \left(m \pi \frac{z}{h_{2}}\right) \cos \left(n \pi \frac{z}{h_{1}}\right) d z .
\end{gathered}
$$

Eq. (46) and (47) represent the approximate continuity conditions at the thickness step. Substitution of (40), (41), (44), and (45) into (46) and (47) leads to:

$$
\begin{gathered}
J_{1}\left(\beta_{2}^{(m)} b\right) A_{2}^{(m)}+Y_{1}\left(\beta_{2}^{(m)} b\right) B_{2}^{(m)}=\Lambda_{m n} J_{1}\left(\beta_{1}^{(n)} b\right) \begin{array}{c}
A_{1}^{(n)} \\
(48)
\end{array} \\
\sum_{m=0}^{\infty}\left(1+\delta_{m 0}\right) \Lambda_{m n} \beta_{2}^{(m)} h_{2}\left[J_{2}\left(\beta_{2}^{(m)} b\right) A_{2}^{(m)}\right. \\
\left.+Y_{2}\left(\beta_{2}^{(m)} b\right) B_{2}^{(m)}\right] \\
=\left(1+\delta_{n 0}\right) \beta_{1}^{(n)} h_{1} J_{2}\left(\beta_{1}^{(n)} b\right) A_{1}^{(n)},
\end{gathered}
$$

where:

$$
\Lambda_{m n}=\frac{2-\delta_{m 0}}{h_{2}} \int_{0}^{h_{2}} \cos \left(m \pi \frac{z}{h_{2}}\right) \cos \left(n \pi \frac{z}{h_{1}}\right) d z .
$$

In addition, a traction-free condition at the outer edge $(r=a)$ requires that:

$$
J_{2}\left(\beta_{2}^{(m)} a\right) A_{2}^{(m)}+Y_{2}\left(\beta_{2}^{(m)} a\right) B_{2}^{(m)}=0 .
$$

Eq. (48), (49), and (51) form a linear system of infinite degrees $(m=0,1,2, \ldots)$. In practice, only a finite subset of the equations may be used to obtain approximate solutions. Let $m$ take values from 0 to $M$. The linear system takes the form:

$$
\Theta \cdot \mathbf{v}=0,
$$

where $\Theta$ is a square matrix of size $2 M+1$ and $\mathbf{v}$ is a vector consisting of all the coefficients, $A_{1}^{(n)}, A_{2}^{(m)}$, and $B_{2}^{(m)}$. For nontrivial solutions, the determinant of the matrix vanishes, namely:

$$
\operatorname{det}[\Theta]=0,
$$

which gives the frequency equation for free vibrations of the stepped plate. The standard procedures of linear analysis then can be used to calculate the resonance frequencies and the corresponding mode shapes.

Similarly, for $h_{1}<h_{2}$, the continuity conditions (46) and (47) become:

$$
\begin{gathered}
u_{\theta 1}^{(n)}=\frac{2-\delta_{n 0}}{h_{1}} \int_{0}^{h_{1}} \sum_{m=0}^{\infty} u_{\theta 2}^{(m)} \cos \left(m \pi \frac{z}{h_{2}}\right) \cos \left(n \pi \frac{z}{h_{1}}\right) d z, \\
\sigma_{r \theta 2}^{(m)}=\frac{2-\delta_{n 0}}{h_{2}} \int_{0}^{h_{1}} \sigma_{r \theta 1}^{(n)} \cos \left(n \pi \frac{z}{h_{1}}\right) \cos \left(m \pi \frac{z}{h_{2}}\right) d z .
\end{gathered}
$$

And (48) and (49) become:

$$
\begin{gathered}
\sum_{m=0}^{\infty} \bar{\Lambda}_{m n}\left[J_{1}\left(\beta_{2}^{(m)} b\right) A_{2}^{(m)}+Y_{1}\left(\beta_{2}^{(m)} b\right) B_{2}^{(m)}\right] \\
=J_{1}\left(\beta_{1}^{(n)} b\right) A_{1}^{(n)}, \quad(56) \\
\left(1+\delta_{m 0}\right) \beta_{2}^{(m)} h_{2}\left[J_{2}\left(\beta_{2}^{(m)} b\right) A_{2}^{(m)}+Y_{2}\left(\beta_{2}^{(m)} b\right) B_{2}^{(m)}\right] \\
=\left(1+\delta_{n 0}\right) \bar{\Lambda}_{m n} \beta_{1}^{(n)} h_{1} J_{2}\left(\beta_{1}^{(n)} b\right) A_{1}^{(n)}, \quad(57)
\end{gathered}
$$

where:

$$
\bar{\Lambda}_{m n}=\frac{2-\delta_{n 0}}{h_{1}} \int_{0}^{h_{1}} \cos \left(m \pi \frac{z}{h_{2}}\right) \cos \left(n \pi \frac{z}{h_{1}}\right) d z .
$$

For $h_{1}=h_{2}$, the two types of continuity conditions converge, and the solution reduces to that for $n$ th-order torsional mode in uniform circular plates.

\section{A. Zeroth-Order Modes $(n=0)$}

Consider first zeroth-order torsional vibrations in the inner region of a stepped plate, for which $n=0$ and the displacement is independent of the thickness coordinate. Such modes also are called radial modes in thin circular plates. The displacement in the outer region in general consists of an infinite series expansion as in (39) in order to satisfy the continuity conditions at the step. Our calculations show that, for the zeroth-order modes at frequencies below the first cut-off frequency, the first term $(m=0)$ in the expansion dominates, and the effect of additional 


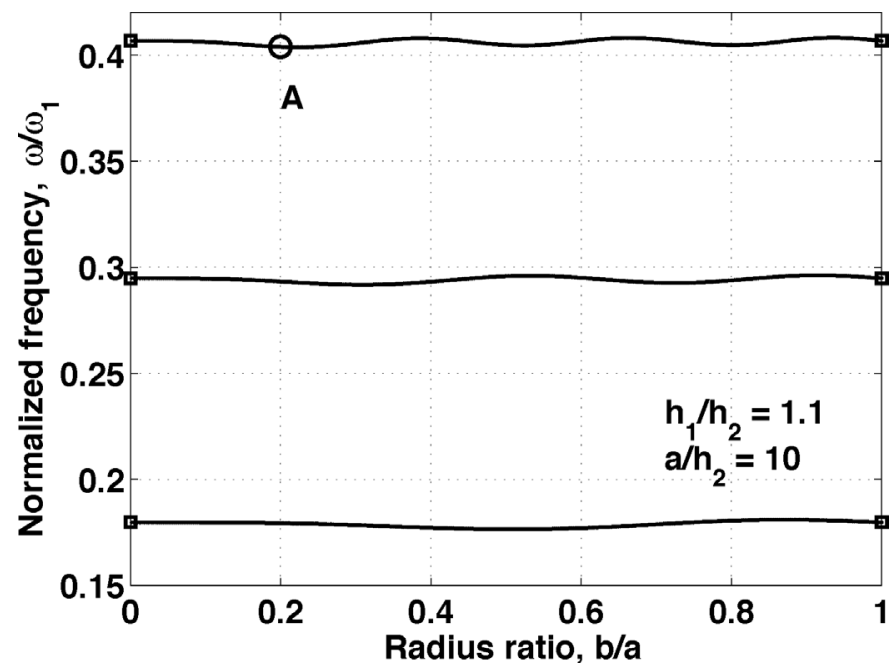

Fig. 4. Frequency spectrum of the zeroth-order torsional vibrations of circular plates with a thickness step $\left(h_{1} / h_{2}=1.1, a / h_{2}=10\right)$. The open squares are the exact solutions for uniform circular plates, independent of the plate thickness. The circle indicates an arbitrarily selected point for the plot of mode shapes in Fig. 5.

terms is negligible. Fig. 4 shows the first three resonance frequencies of the zeroth-order modes varying with the radius of the inner region. The frequencies are normalized by the cut-off frequency, $\omega_{1}=\left(\pi / h_{1}\right) \sqrt{\mu / \rho}$. The thickness ratio is fixed as $h_{1} / h_{2}=1.1$, and the outer radius is $a / h_{2}=10$. At both ends of the plot with the radius ratio $b / a$ being 0 or 1 , the stepped plate reduces to uniform circular plates with thickness $h_{2}$ and $h_{1}$, respectively. The zeroth-order resonance frequencies of a uniform circular plate are given by (19) with $n=0$ and labeled in Fig. 4 as open squares. It is interesting to note that, although the zeroth-order resonance frequencies of a uniform circular plate are independent of its thickness and thus identical at both ends of Fig. 4, the frequencies oscillate slightly in between, with the number of oscillations increasing with the mode number and the amplitude of oscillation depending on the thickness of the step. Such oscillation may be caused by the interactions between the torsional waves and the discontinuous boundary at the step.

To check the continuity conditions at the step, Fig. 5 plots the mode shape corresponding to an arbitrarily selected point labeled as A $(b / a=0.2)$ in Fig. 4. The zeroth-order two-dimensional components of the displacement and the traction are plotted, both normalized by the maximum absolute values. It is noted that the displacement is continuous across the step, but the stress by itself is not continuous. The error is due to the approximation of the traction continuity condition specified by (47), which leads to continuity of the total traction for the zeroth-order modes, i.e., $\sigma_{r \theta 1}^{(0)} h_{1}=\sigma_{r \theta 2}^{(0)} h_{2}$ at $r=b$. Such approximation is reasonable for the zeroth-order modes. Also noted is that the variations of the displacement and the stress along the thickness direction are different for the inner and outer regions due to the thickness step. The error, however, is small when the thickness ratio is close to one.
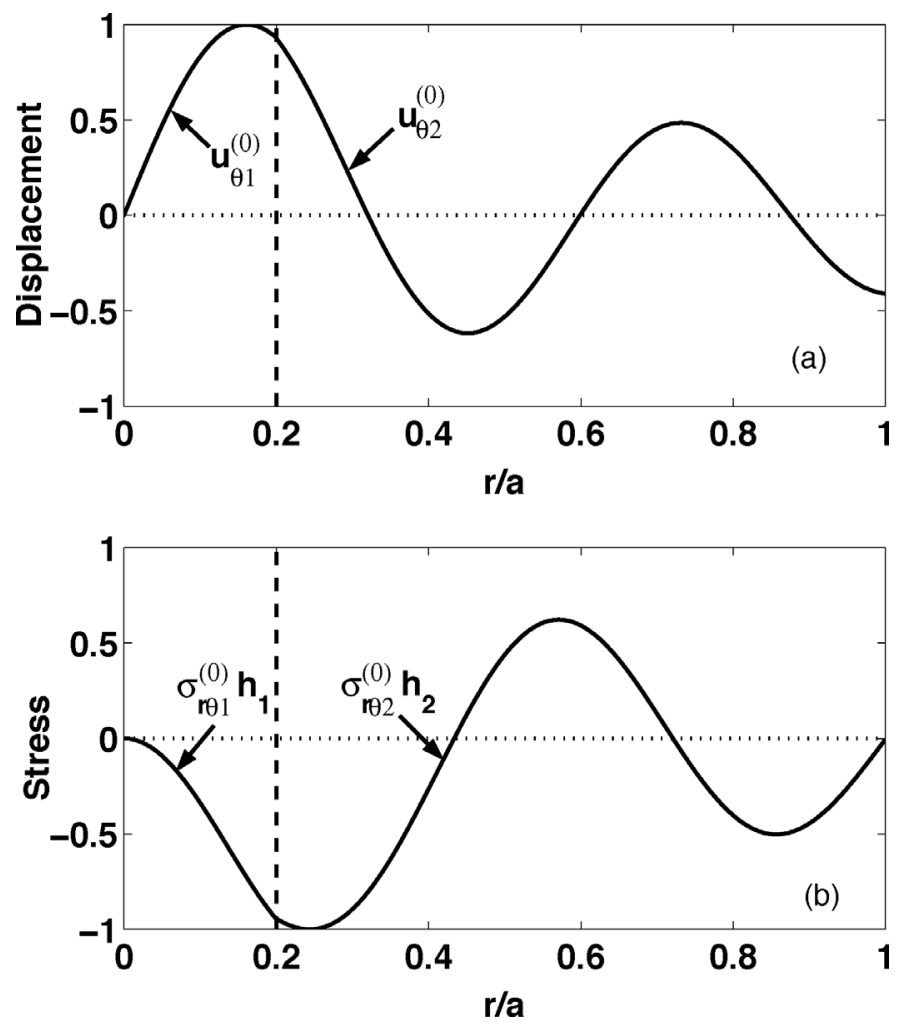

Fig. 5. Mode shapes for the zeroth-order torsional mode corresponding to point $\mathrm{A}(b / a=0.2)$ in Fig. 4 , in a circular plate with a thickness step $\left(h_{1} / h_{2}=1.1, a / h_{2}=10\right)$. The dashed line indicates the location of the thickness step.

\section{B. First-Order Modes $(n=1)$}

Next consider the first-order modes with $n=1$. The focus is on the vibration modes near the first cut-off frequency. A convergence study with up to five terms shows that, in this case, the second term $(m=1)$ dominates in the series expansion (39) for the displacement of the outer region. Including other terms produces negligible difference in the results. Fig. 6 shows the resonance frequencies varying with the radius ratio $b / a$ for a fixed thickness ratio $h_{1} / h_{2}=1.1$ and outer radius $a / h_{2}=10$. The left end of the plot corresponds to a uniform circular plate of thickness $h_{2}$, which has a cut-off frequency, $\omega_{c 2}=\left(\pi / h_{2}\right) \sqrt{\mu / \rho}$, and the right end corresponds to a uniform plate of thickness $h_{1}$ with a lower cut-off frequency, $\omega_{c 1}=\left(\pi / h_{1}\right) \sqrt{\mu / \rho}$ $\left(h_{1}>h_{2}\right)$. The exact solutions for the uniform circular plates are calculated from (19) with $n=1$ and labeled as squares at both ends. In between, the resonance frequencies change continuously. No first-order modes can be found below the cut-off frequency $\omega_{c 1}$. Of particular interest are the vibrational modes with frequencies between the two cut-off frequencies. For these modes, the radial wave number in the outer region, $\beta_{2}^{(1)}$, is imaginary, and the Bessel functions describing the distributions of the displacement and the stress asymptotically reduce to those decaying exponentially from the step. Consequently, the vibration is trapped within the inner region. Fig. 7 plots the mode shape associated with the point $\mathrm{A}(b / a=0.2)$ 


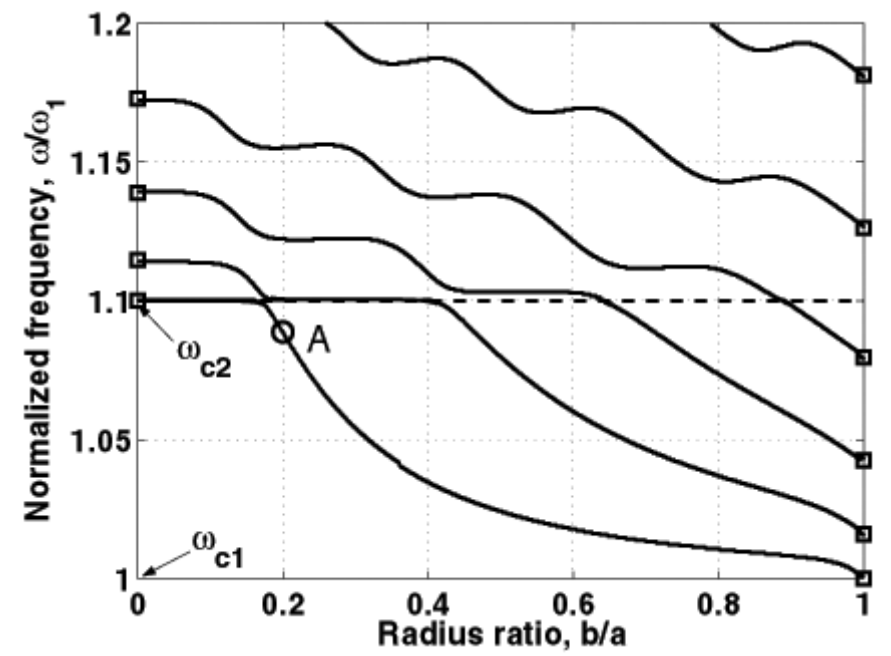

Fig. 6. Frequency spectrum of the first-order torsional vibrations of circular plates with a thickness step $\left(h_{1} / h_{2}=1.1, a / h_{2}=10\right)$. The open squares are the exact solutions for uniform circular plates. The circle indicates an arbitrarily selected point for the plot of mode shapes in Fig. 7.

in Fig. 6. From (46) and (47), the approximate continuity conditions at the step $(r=b)$ for the first-order modes are: $u_{\theta 2}^{(1)}=\Lambda_{11} u_{\theta 1}^{(1)}$ and $\sigma_{r \theta 1}^{(1)} h_{1}=\Lambda_{11} \sigma_{r \theta 2}^{(1)} h_{2}$, where $\Lambda_{11}=0.939$. Therefore, the continuity conditions are approximately satisfied. As shown in Fig. 7, both the displacement and the stress decay exponentially from the step, which confirms the existence of trapped torsional modes in the stepped plate.

Similar analyses can be conducted for higher-order modes (e.g., $n=2,3, \ldots$ ), and trapped torsional modes are expected to exist near each cut-off frequency.

\section{Trapped Torsional Modes in Infinite Plates}

The existence of trapped torsional modes in stepped plates offers possibilities to design an array of localized energy traps (or resonant cavities) on a large plate, each serving as a torsional-mode resonator. These resonators are very sensitive to surface loading and may be used for a variety of sensing applications. Each thickness step can be a circular mesa, for example, formed by machining, etching, or bonding of a decal onto the surface of the plate. The design parameters include the thickness and the radius of the mesa as well as the spacing between adjacent mesas. The frequencies of the trapped modes depend on the mesa dimensions, and the spacing must be large enough to avoid coupling between adjacent resonators. Each mesa thus can be considered sitting on an infinite plate $(a \rightarrow \infty)$ isolated from the others. In this case, it is more convenient to use Hankel functions to describe the displacement outside the mesa, and by their asymptotic behavior only the first Hankel function remains so that the displacement at the infinite outer boundary vanishes. Therefore, any possible modes must be trapped near the mesa. Fig. 8 shows the resonance frequencies for the first-order modes
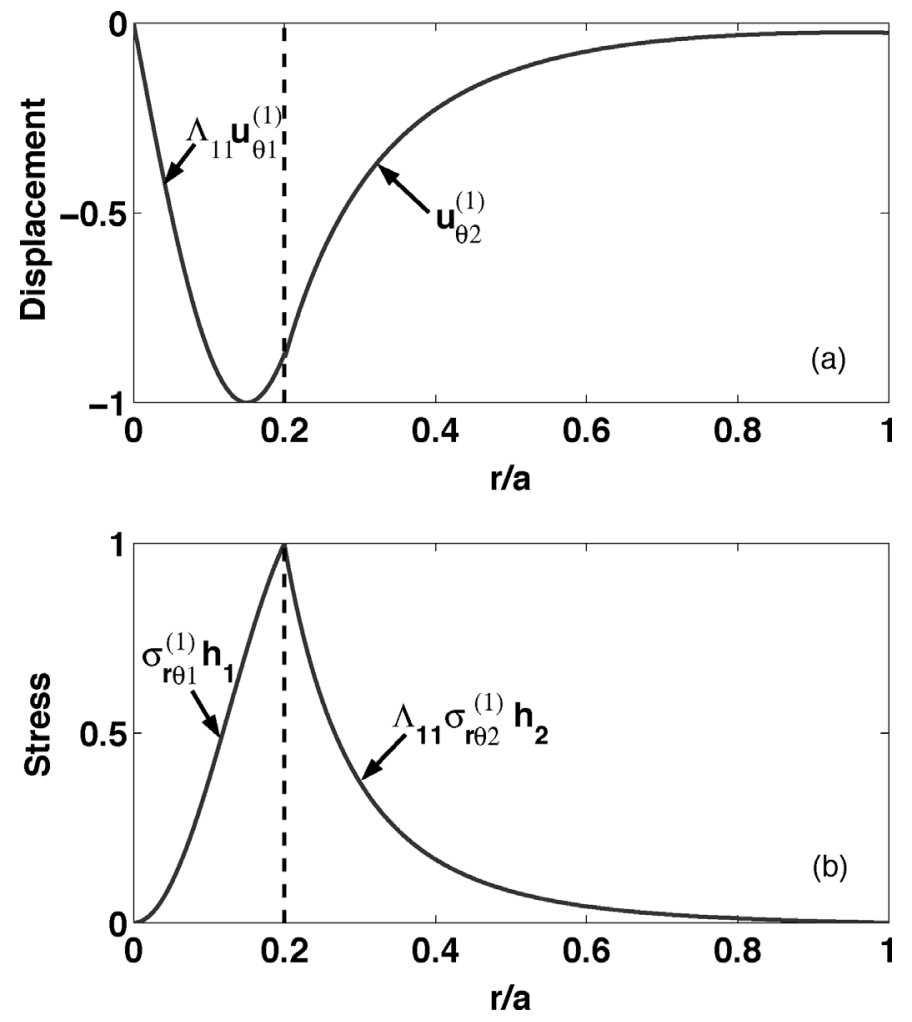

Fig. 7. Mode shapes of the first-order mode corresponding to point A of Fig. 6, showing the characteristic of a trapped torsional mode in a circular plate with a thickness step $\left(h_{1} / h_{2}=1.1, a / h_{2}=10\right)$. The dashed line indicates the location of the step.

varying with the mesa radius for a fixed thickness ratio $\left(h_{1} / h_{2}=1.1\right)$. It is found that, depending on the mesa dimensions, there may exist zero, one, or multiple trapped modes. Fig. 9 depicts a diagram for the number of trapped first-order torsional modes in a circular mesa with the radius $b / h_{2}$ and the thickness ratio $h_{1} / h_{2}$ as the coordinates. The lines divide the plane into six regions, with the number of trapped modes denoted in each region. The diagram is independent of the material properties as long as the plate is homogeneous, isotropic, and elastic. The diagram may be read in two ways. For a circular mesa with a given thickness, there exists a critical radius, below which no trapped mode exists; multiple trapped modes may exist when the radius is large. It is often desirable to have a single trapped mode, which requires a mesa radius within the window bounded by the lowest two lines in Fig. 9. Alternatively, if the mesa radius is fixed, there exists a critical thickness, below which no trapped modes can be found, and a single trapped mode exists when the thickness is within the window. Such a diagram may serve as a guide for designing energy-trapped torsional-mode resonators.

The theoretical results from this study are compared to experimental measurements. A brief description of the experimental procedure follows. More details will be presented elsewhere [26]. Circular mesas were machined in a 6000 series cast aluminum plate, with the mesa perimeters defined by removing metal in the form of a moat. The moat was made wide enough (typically exceeding two 
TABLE I

Dimensions of Six Samples Used in Experimental Measurements.

\begin{tabular}{lcccccc}
\hline & $\mathrm{A}$ & $\mathrm{B}$ & $\mathrm{C}$ & $\mathrm{D}$ & $\mathrm{E}$ & $\mathrm{F}$ \\
\hline Diameter, $2 b(\mathrm{~mm})$ & 37.668 & 37.846 & 37.719 & 25.908 & 25.654 & 25.654 \\
Thickness, $h_{1}(\mathrm{~mm})$ & 3.1369 & 3.0683 & 3.0607 & 3.1679 & 3.1496 & 3.1242 \\
Thickness, $h_{2}(\mathrm{~mm})$ & 2.9464 & 2.6416 & 3.0226 & 2.6797 & 2.9972 & 2.8702 \\
Thickness ratio, $h_{1} / h_{2}$ & 1.06 & 1.16 & 1.01 & 1.18 & 1.05 & 1.09 \\
Ratio $b / h_{2}$ & 6.39 & 7.16 & 6.24 & 4.83 & 4.28 & 4.47 \\
\hline
\end{tabular}

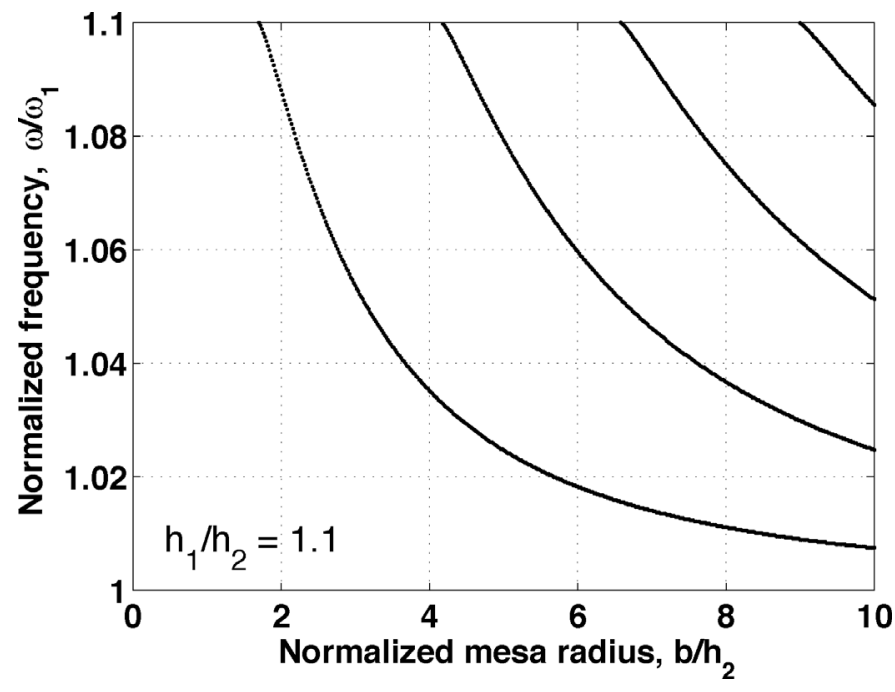

Fig. 8. The resonant frequencies of trapped first-order torsional modes in a circular mesa on an infinite plate $\left(h_{1} / h_{2}=1.1\right)$.

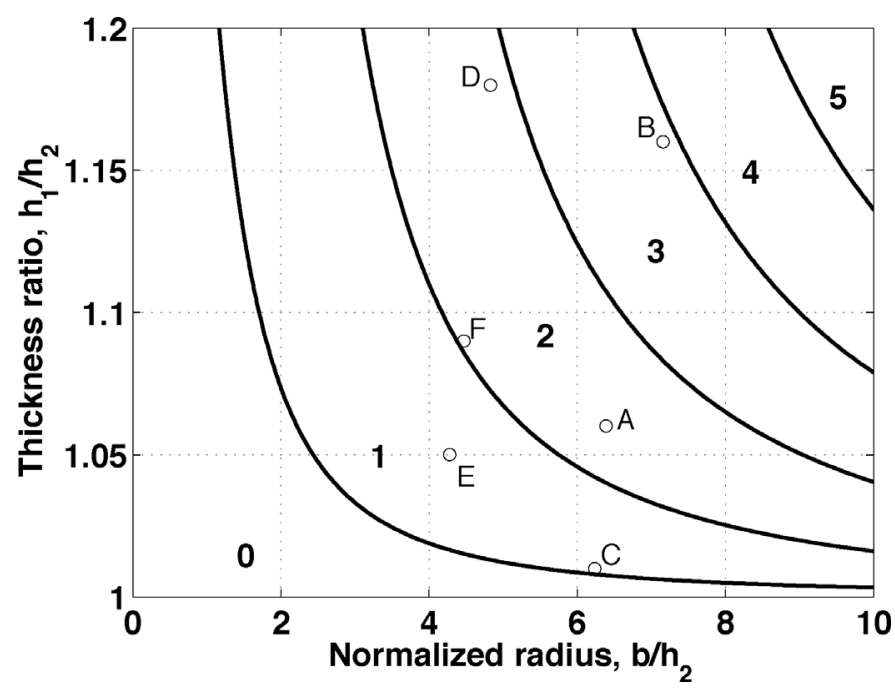

Fig. 9. A diagram for the number of trapped first-order torsional modes in a circular mesa on an infinite plate. The circles correspond to the samples $\mathrm{A}-\mathrm{F}$ used in experiments.

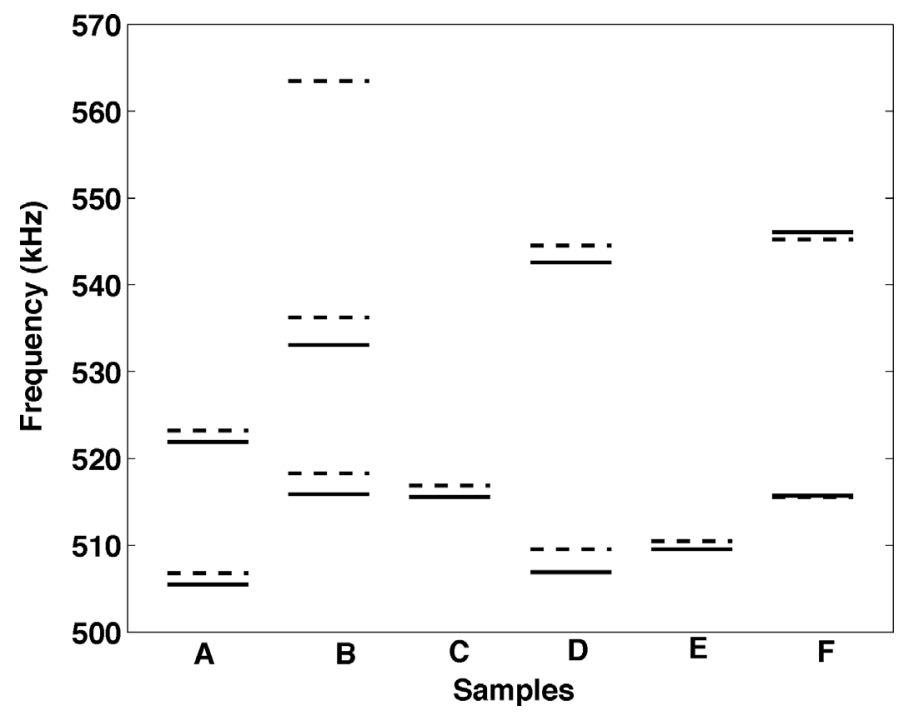

Fig. 10. Comparison of resonance frequencies of trapped torsional modes between experiments (solid lines) and predictions (dashed lines). The dimensions of the samples A-F are listed in Table I and indicated in Fig. 9.

or more wavelengths) so that the displacement, which decayed exponentially away from the mesa perimeter, was negligible at the outer edge of the moat and hence each mesa was isolated acoustically. A noncontact electromagnetic acoustic transducer (EMAT) was used to generate oscillatory surface tractions. Torsional modes were excited when the traction force was applied in the circumferential direction. Resonant frequencies were determined by adjusting the pulse train frequency for peak initial amplitude as observed with an oscilloscope. The system error of this measurement is estimated to be $5 \%$. A series of tests were conducted to measure the surface motion at resonances in order to confirm the observation of trapped torsional modes, for example, by using an absorbing stylus to determine the locations of displacement maxima and using a pick-up coil to confirm the circumferential motion. Table I lists the dimensions of six circular mesas used in experiments. These samples correspond to the points $\mathrm{A}-\mathrm{F}$ labeled in Fig. 9. The diagram predicts that samples $\mathrm{C}$ and E have a single trapped torsional mode. Samples A, D, and F have two trapped modes. Sample B has three trapped modes. Fig. 10 shows the measured resonance frequencies in comparison with the predictions by the approximate method developed in this paper. In calculating the fre- 
quencies, the shear modulus and the mass density of the cast aluminum are taken to be $26 \mathrm{GPa}$ and $2700 \mathrm{~kg} / \mathrm{m}^{3}$, respectively. The agreement between the measurements and the predictions is excellent, except for Sample B, in which only two trapped modes were observed but three were predicted by the theory. It was noted in experiments that spurious modes were generated and not trapped when the step thickness was relatively large and the frequency was high. The validity of the approximate theory is thus limited to small step thickness with frequencies close to the cut-off frequency.

\section{Conclusions}

This paper presents a theoretical study of torsional vibrations in isotropic elastic plates. In particular, an approximate method is developed to analyze torsional vibrations in circular plates with thickness steps. Approximate solutions are presented for the zeroth- and first-order torsional modes. Of practical interest is the trapped firstorder mode, which is theoretically predicted and confirmed by the mode shapes. The number of trapped first-order torsional modes in a circular mesa on an infinite plate is determined as a function of the normalized geometric parameters, which may serve as a guide for designing distributed torsional-mode resonators for sensing applications. Comparisons between the theoretical predictions and experimental measurements show close agreements in the resonance frequencies of trapped torsional modes.

\section{ACKNOWLEDGMENT}

R. H. acknowledges College of Engineering at The University of Texas, Austin, for financial support.

\section{REFERENCES}

[1] H. G. Craighead, "Nanoelectromechanical systems," Science, vol. 290, pp. 1532-1535, 2000.

[2] A. M. Fennimore, T. D. Yuzvinsky, W.-Q. Han, M. S. Fuhrer, J. Cumings, and A. Zettl, "Rotational actuators based on carbon nanotubes," Nature, vol. 424, pp. 408-410, 2003.

[3] P. A. Williams, S. J. Papadakis, A. M. Patel, M. R. Falvo, S. Washburn, and R. Superfine, "Torsional responses and stiffening of individual multiwalled carbon nanotubes," Phys. Rev. Lett., vol. 89, no. 25, pp. 255502-1-255502-4, 2002.

[4] P. A. Williams, S. J. Papadakis, A. M. Patel, M. R. Falvo, S. Washburn, and R. Superfine, "Fabrication of nanometer-scale mechanical devices incorporating individual multiwalled carbon nanotubes as torsional springs," Appl. Phys. Lett., vol. 82, pp. 805-807, 2003.

[5] D. W. Carr, S. Evoy, L. Sekaric, H. G. Craighead, and J. M. Parpia, "Measurement of mechanical resonance and losses in nanometer scale silicon wires," Appl. Phys. Lett., vol. 75, pp. 920-922, 1999.

[6] D. J. Gorman, Free Vibration Analysis of Beams and Shafts. New York: Wiley, 1975, pp. 186-222.

[7] A. W. Leissa and J. So, "Comparisons of vibration frequencies for rods and beams from one-dimensional and three-dimensional analysis," J. Acoust. Soc. Amer., vol. 98, pp. 2122-2135, 1995.
[8] J. Q. Pan, J. Pan, R. S. Ming, and T. Lin, "Three-dimensional response and intensity of torsional vibrations in a stepped shaft," J. Sound Vib., vol. 236, pp. 115-128, 2000.

[9] Y. Y. Kim and J. H. Kim, "Stress analysis of a stepped shaft under remote torsion," Q. J. Mechan. Appl. Math., vol. 48, pp. $517-530,1995$.

[10] T. Tsuji, N. Noda, T. Shibuya, and T. Koizumi, "Stress singularity in torsion problem for bonded bars," Int. J. Solids Struct., vol. 27, pp. 1059-1071, 1991.

[11] W. Johnson, B. A. Auld, E. Segal, and F. Passarelli, "Trapped torsional modes in solid cylinders," J. Acoust. Soc. Amer., vol. 100, pp. 285-293, 1996.

[12] R. Bechmann, "Quartz AT-type filter crystals for the frequency range 0.7 to 60 Mc.," in Proc. Inst. Radio Eng., vol. 49, 1961, pp. 523-524.

[13] W. Shockley, D. R. Curran, and D. J. Koneval, "Energy trapping and related studies of multiple electrode filter crystals," in Proc. 17th Annu. Freq. Contr. Symp., 1963, pp. 88-126.

[14] R. D. Mindlin and P. C. Y. Lee, "Thickness-shear and flexural vibrations of partially plated, crystal plates," Int. J. Solids Struct., vol. 2, pp. 125-139, 1966.

[15] H. F. Tiersten, "Analysis of trapped-energy resonators operating in overtones of coupled thickness shear and thickness twist," $J$. Acoust. Soc. Amer., vol. 59, pp. 879-888, 1976.

[16] P. C. Y. Lee, C. Zee, and C. A. Brebbia, "Thickness shear thickness twist, and flexural vibrations of rectangular AT-cut quartz plates with patch electrodes," J. Acoust. Soc. Amer., vol. 72, pp. 1855-1862, 1982.

[17] H. Sekimoto, Y. Watanabe, and M. Nakazawa, "Forced vibrations of thickness-flexure, face-shear and face-flexure in rectangular AT-cut quartz plates," in Proc. 46th Annu. Freq. Contr. Symp., 1992, pp. 532-536.

[18] P. C. Y. Lee and J. Wang, "Piezoelectrically forced thicknessshear and flexural vibrations of contoured quartz resonators," $J$. Appl. Phys., vol. 79, pp. 3411-3422, 1996.

[19] J. Wang, P. C. Y. Lee, and D. H. Bailey, "Thickness-shear and flexural vibrations of linearly contoured crystal strips with multiprecision computation," Comput. Struct., vol. 70, pp. 437-445, 1999.

[20] A. E. H. Love, Mathematical Theory of Elasticity. New York: Cambridge Univ. Press, 1927, p. 497.

[21] M. Onoe, "Contour vibrations of isotropic circular plates," $J$. Acoust. Soc. Amer., vol. 28, pp. 1158-1162, 1956.

[22] A. H. Meitzler, "An ultrasonic technique for determining the elastic constants of glass wafers," in Proc. IEEE Ultrason. Symp., 1992, pp. 963-968.

[23] S. K. Clark, "Torsional wave propagation in hollow cylindrical bars," J. Acoust. Soc. Amer., vol. 28, pp. 1163-1165, 1956.

[24] K. F. Graff, Wave Motion in Elastic Solids. New York: Dover, 1991, pp. $468-470$.

[25] J.-H. Kang, "Three-dimensional vibration analysis of thick, circular and annular plates with nonlinear thickness variation," Comput. Struct., vol. 81, pp. 1663-1675, 2003.

[26] T. Knowles, M. K. Kang, and R. Huang, "Trapped torsional vibrations in elastic plates," Appl. Phys. Lett., vol. 87, 201911, 2005.

[27] D. Zhou, F. T. K. Au, Y. K. Cheung, and S. H. Lo, "Threedimensional vibration analysis of circular and annular plates via the Chebyshev-Ritz method," Int. J. Solids Struct., vol. 40, pp. 3089-3105, 2003.

[28] R. D. Mindlin, An Introduction to the Mathematical Theory of Vibrations of Elastic Plates. A Monograph Prepared for U.S. Army Signal Corps Engineering Laboratories, Fort Monmouth, NJ 1955.

[29] R. D. Mindlin, "High frequency vibrations of crystal plates," $Q$. Appl. Math., vol. 19, pp. 51-61, 1961.

[30] H. F. Tiersten, Linear Piezoelectric Plate Vibrations. New York: Plenum, 1969, pp. 129-168.

[31] P. C. Y. Lee, S. Syngellakis, and J. P. Hou, "A two-dimensional theory for high-frequency vibrations of piezoelectric crystal plates with or without electrodes," J. Appl. Phys., vol. 61, pp. 1249-1262, 1987.

[32] R. D. Mindlin and M. Forray, "Thickness-shear and flexural vibrations of contoured crystal plates," J. Appl. Phys., vol. 25, pp. 12-20, 1954. 


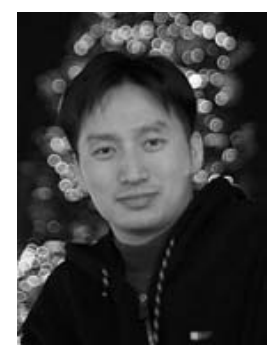

Min Kyoo Kang was born in Taegu, South Korea, in 1976. He received his B.S. degree in the School of Mechanical Engineering of Hanyang University in South Korea in 2000 and received M.S. degree in machine design and production engineering from the same university in 2002. Currently he is a Ph.D. student in the department of Aerospace Engineering and Engineering Mechanics of The University of Texas at Austin. His research interests include vibrations and wave propagation in beams and plates, piezoelectric devices, and molecular dynamics simulations.

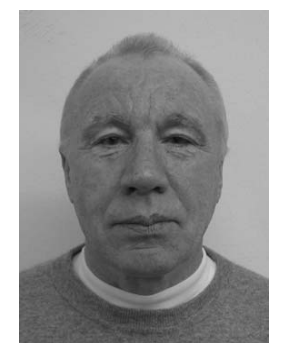

Terence Knowles (M'00) received his B.S. degree in Applied Physics from the University of London in 1965, and his M.S. in Physics from De Paul University, Chicago, IL in 1970. He is co-founder and president of Texzec Inc. at Round Rock, TX. His research interests include vibrations and wave propagation in beams, rods, and plates, ultrasonic transducers, and sensors. He holds numerous patents in these areas.

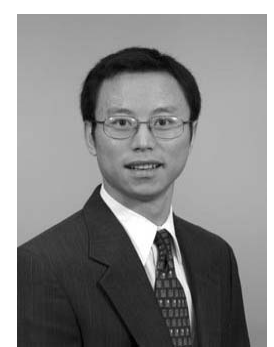

Rui Huang received his B.S. degree in Theoretical and Applied Mechanics from the University of Science and Technology of China in 1994 and his Ph.D. degree in Civil and Environmental Engineering, with specialty in Mechanics, Materials, and Structures, from Princeton University in 2001. He is currently an assistant professor of Aerospace Engineering and Engineering Mechanics at the University of Texas at Austin. His research interests include mechanics and materials in microelectromechanical systems (MEMS), microelectronics, and emerging nanotechnologies. 